\title{
Hybrid Differential Evolution - Particle Swarm Optimization Algorithm for Solving Global Optimization Problems
}

\author{
${ }^{1}$ Millie Pant, ${ }^{1}$ Radha Thangaraj, ${ }^{2}$ Crina Grosan and ${ }^{3}$ Ajith Abraham \\ ${ }^{1}$ Department. of Paper Technology, IIT Roorkee, India \\ ${ }^{2}$ Babes - Bolyai University, Cluj-Napaco, Romania \\ ${ }^{3}$ Center of Excellence for Quantifiable Quality of Service, \\ Norwegian University of Science and Technology, Norway \\ millifpt@iitr.ernet.in, t.radha@ieee.org, cgrosan@cs.ubbcluj.ro, ajith.abraham@ieee.org
}

\begin{abstract}
This paper presents a simple, hybrid two phase global optimization algorithm called DE-PSO for solving global optimization problems. DE-PSO consists of alternating phases of Differential Evolution (DE) and Particle Swarm Optimization (PSO). The algorithm is designed so as to preserve the strengths of both the algorithms. Empirical results show that the proposed DE-PSO is quite competent for solving the considered test functions as well as real life problems.
\end{abstract}

\section{Introduction}

In the past few years, DE and PSO have emerged as powerful optimization tools for solving complex optimization problems. Both are population based stochastic search techniques inspired by nature. Although, DE and PSO have been successfully applied to a wide range of problems including test and real life problems both have certain shortcomings associated with them. The major problems being lack of diversity resulting in a suboptimal solution [1] or a slow convergence rate. In order to improve the performance of these algorithms a number of variations have been suggested in literature, one of them being the hybridization of the two algorithms. Some hybrid versions of DE and PSO include Hendtlass approach [2], where the population evolved by DE is optimized by using PSO, Kannan approach [3]; in which DE is applied to each particle for a finite number of iterations to determine the best particle which is then included into the population. Methods of Zhang and Xie [4] and Talbi and Batauche [5] apply DE to the best particle obtained by PSO. Omran et al [6] developed a hybrid version consisting of Barebones PSO and DE. In Zhi Feng et al [7] hybrid version, the candidate solution is generated either by DE or by PSO according to some fixed probability distribution.

In this paper we propose a simple hybrid version of DE and PSO, called DE-PSO. DE-PSO starts with the usual DE and incorporated PSO to reach to the optimal solution.

The global optimization problems in this paper follow the form:

Min $\mathrm{f}(\mathrm{X})$ subject to $\mathrm{X} \in \Lambda$

where $\mathrm{X}$ is a continuous variable vector with domain $\Lambda \in \mathrm{R}^{\mathrm{n}}$. The domain $\Lambda$ is defined within upper and lower limits of each dimension. We notate the global optimal solution by $\mathrm{X}^{*}$, with its corresponding global optimal function value $\mathrm{f}\left(\mathrm{X}^{*}\right)$.

The remaining of the paper is organized as follows: in Sections 2 and 3, we give a brief description of DE and PSO algorithms respectively. Section 4, describes the proposed DE-PSO algorithm. Benchmark problems, some real world problems and corresponding numerical results are given in Section 5. The paper finally concludes with Section 6.

\section{Differential Evolution}

Differential evolution (DE) is an Evolutionary Algorithm (EA) proposed by Storn and Price in 1995 [8]. DE is similar to other EAs particularly Genetic Algorithms (GA) [9] in the sense that it uses the same evolutionary operators like selection recombination and mutation like that of GA. However the significant difference is that DE uses distance and direction information from the current population to guide the search process. The performance of DE depends on the 
manipulation of target vector and difference vector in order to obtain a trial vector. Mutation is the main operator in DE. A brief working may be described as: For a D-dimensional search space, each target vector $x_{i, g}$, a mutant vector is generated by

$$
v_{i, g+1}=x_{r_{1}, g}+F *\left(x_{r_{2}, g}-x_{r_{3}, g}\right)
$$

where $r_{1}, r_{2}, r_{3} \in\{1,2, \ldots ., N P\}$ are randomly chosen integers, must be different from each other and also different from the running index i. $F(>0)$ is a scaling factor which controls the amplification of the differential evolution $\left(x_{r_{2}, g}-x_{r_{3}, g}\right)$. In order to increase the diversity of the perturbed parameter vectors, crossover is introduced [9]. The parent vector is mixed with the mutated vector to produce a trial vector $u_{j i, g+1}$, $u_{j i, g+1}=\left\{\begin{array}{lll}v_{j i, g+1} & \text { if }\left(\text { rand }_{j} \leq C R\right) & \text { or } \quad\left(j=j_{\text {rand }}\right) \\ x_{j i, g} & \text { if }\left(\text { rand }_{j}>C R\right) & \text { and }\left(j \neq j_{\text {rand }}\right)\end{array}\right.$ where $\mathrm{j}=1,2, \ldots \ldots, \mathrm{D} ;$ rand $_{j} \in[0,1] ; \mathrm{CR}$ is the crossover constant takes values in the range $[0,1]$ and $j_{\text {rand }} \in(1,2, \ldots \ldots, D)$ is the randomly chosen index.

Selection is the step to choose the vector between the target vector and the trial vector with the aim of creating an individual for the next generation.

\section{Particle Swarm Optimization}

PSO was proposed in 1995 by Kennedy and Eberhart [10]. The mechanism of PSO is inspired from the complex social behavior shown by the natural species. For a D-dimensional search space the position of the ith particle is represented as $\mathrm{X}_{\mathrm{i}}=\left(\mathrm{x}_{\mathrm{i} 1}, \mathrm{X}_{\mathrm{i} 2}, \ldots \mathrm{x}_{\mathrm{iD}}\right)$. Each particle maintains a memory of its previous best position $\mathrm{P}_{\mathrm{i}}=\left(\mathrm{p}_{\mathrm{i} 1}, \mathrm{p}_{\mathrm{i} 2} \ldots \mathrm{p}_{\mathrm{iD}}\right)$ and a velocity $\mathrm{V}_{\mathrm{i}}=\left(\mathrm{v}_{\mathrm{i} 1}\right.$, $\left.\mathrm{v}_{\mathrm{i} 2}, \ldots \mathrm{v}_{\mathrm{iD}}\right)$ along each dimension . At each iteration, the $\mathrm{P}$ vector of the particle with best fitness in the local neighborhood, designated $\mathrm{g}$, and the $\mathrm{P}$ vector of the current particle are combined to adjust the velocity along each dimension and a new position of the particle is determined using that velocity. The two basic equations which govern the working of PSO are that of velocity vector and position vector are given by:

$$
\begin{aligned}
v_{i d} & =\omega v_{i d}+c_{1} r_{1}\left(p_{i d}-x_{i d}\right)+c_{2} r_{2}\left(p_{g d}-x_{i d}\right) \\
x_{i d} & =x_{i d}+v_{i d}
\end{aligned}
$$

The first part of equation (2) represents the inertia of the previous velocity, the second part is tells us about the personal thinking of the particle and the third part represents the cooperation among particles and is therefore named as the social component. Acceleration constants c1, c2 and inertia weight $\omega$ are predefined by the user and $\mathrm{r} 1, \mathrm{r} 2$ are the uniformly generated random numbers in the range of $[0,1]$.

\section{Proposed DE-PSO}

The proposed DE-PSO as mentioned earlier is a hybrid version of DE and PSO. DE-PSO starts like the usual DE algorithm up to the point where the trial vector is generated. If the trial vector satisfies the conditions given by equation (4), then it is included in the population otherwise the algorithm enters the PSO phase and generates a new candidate solution. The method is repeated iteratively till the optimum value is reached. The inclusion of PSO phase creates a perturbation in the population, which in turn helps in maintaining diversity of the population and producing a good optimal solution.

The pseudo code of the Hybrid DE and PSO (DE-PSO) Algorithm is:

Initialize the population

For $\mathrm{i}=1$ to $\mathrm{N}$ (Population size) do

Select $r_{1}, r_{2}, r_{3} \in N$ randomly

$/ / r_{1}, r_{2}, r_{3}$ are selected such that $r_{1} \neq r_{2} \neq r_{3} / /$

For $\mathrm{j}=1$ to $\mathrm{D}$ (dimension) do

Select $\mathrm{j}_{\text {rand }} \in \mathrm{D}$

If (rand ()$<\mathrm{CR}$ or $\mathrm{j}=\mathrm{j}_{\text {rand }}$ )

// rand () denotes a uniformly distributed random number between 0 and $1 / /$

$$
U_{j i, g+1}=x_{r_{1}, g}+F *\left(x_{r_{2}, g}-x_{r_{3}, g}\right)
$$

End if

If $\left(f\left(U_{j i, g+1}\right)<f\left(X_{j i, g}\right)\right)$ then

$X_{j i, g+1}=U_{j i, g+1}$

Else

PSO activated

Find a new particle using equations (2) and (3).

(Let this particle be $T X_{j i}$ )

If $\left(f\left(T X_{j i}\right)<f\left(X_{j i, g}\right)\right)$ then $X_{j i, g+1}=T X_{j i}$

Else $X_{j i, g+1}=X_{j i, g}$

End if

End if

End for

End for. 


\section{Experimental Settings}

Experimental settings for proposed DE-PSO, DE and PSO (Table 2):

For dimension 30: $P o p=30$, run=30, Max Gne $=3000$

For dimension 50: $\mathrm{Pop}=50$, run=20, Max Gne $=5000$

For dimension 100: $\quad P o p=100, \quad r u n=10, \quad$ Max

Gne $=10000$

Experimental settings for proposed DE-PSO and DEPSO [7] (Table 4):

Pop: 30, dim: 30, Max Gne.: 12000

Experimental settings for proposed DE-PSO and BBDE [6] (Table 4):

Pop: 30, dim: 30, Max number of function evaluations: 100000

In the above mentioned settings, Pop denotes the population size taken; run denotes the number of times an algorithm is executed; Max Gne denotes the maximum number of generations allowed for each algorithm

Computer Settings:

All the three algorithms were implemented using Turbo C++ on a PC compatible with Pentium IV, a 3.2 $\mathrm{GHz}$ processor and $2 \mathrm{~GB}$ of RAM.

\subsection{Benchmark Problems and Results}

For the present study, a set of twelve unconstrained benchmark problems is taken (Table 1). Although this collection may not be called exhaustive but it is a good launch pad to decide the authenticity of an optimization algorithm. All the problems are scalable and are tested for dimensions 30, 50, 100. Functions f1, f3, f5-f9 are highly modal where the complexity of the problem increases with the increase in the number of variables. We have also taken a noisy function (f9), where a uniformly distributed random noise is added to the function. $\mathrm{f} 11$ is a discontinuous function and for $\mathrm{f} 12$, the optimum lies in a plateau like region.

Numerical results in Table 2 show the performance of DE-PSO with the classical PSO and DE. In Table 4, we give a comparison of DE-PSO with two other recently proposed hybrid version of DE and PSO, namely BBDE [6] and DEPSO [7].

Table 2 shows that the proposed DE-PSO algorithm gives a superior performance in comparison to both $\mathrm{DE}$ and PSO in almost all the test cases. Table 3 gives the number of times PSO phase is activated so as to reach the optimal solution. In [6] and [7] problems of dimension 30 are taken. For the purpose of comparison we took the problems common in [6] and [7] and followed the same experimental settings. Numerical results in Table 4 show that in comparison to [7], DE-
PSO gave a better performance in four out of five test cases tied and in comparison to BBDE, DE-PSO gave a superior performance in three out of five test cases tried. For f12 both BBDE and DE-PSO gave same performance.

\subsection{Real life Problems and Results}

The credibility of an optimization algorithm also depends on its ability to solve real life problems. In this paper we took three real life problems to validate the performance of the proposed DE-PSO.

Gas transmission compressor design [11]:

$$
\begin{aligned}
& \operatorname{Min} f(x)=8.61 \times 10^{5} \times x_{1}^{1 / 2} x_{2} x_{3}^{-2 / 3}\left(x_{2}{ }^{2}-1\right)^{-1 / 2} \\
& +3.69 \times 10^{4} \times x_{3}+7.72 \times 10^{8} \times x_{1}^{-1} x_{2}^{0.219} \\
& -765.43 \times 10^{6} \times x_{1}^{-1}
\end{aligned}
$$

Subject to: $10 \leq x_{1} \leq 55,1.1 \leq x_{2} \leq 2,10 \leq x_{3} \leq 40$

Optimal thermohydralic performance of an artificially roughened air heater [12]:

$\operatorname{Max} L=2.51 * \ln e^{+}+5.5-0.1 R_{M}-G_{H}$

Where $R_{M}=0.95 x_{2}^{0.53} ; G H=4.5\left(e^{+}\right)^{0.28}(0.7)^{0.57}$;

$e^{+}=x_{1} x_{3}(\bar{f} / 2)^{1 / 2} ; \bar{f}=\left(f_{s}+f_{r}\right) / 2 ; f_{s}=0.079 x_{3}{ }^{-0.25}$;

$f_{r}=2\left(0.95 x_{3}{ }^{0.53}+2.5 * \ln \left(1 / 2 x_{1}\right)^{2}-3.75\right)^{-2}$;

Subject to: $0.02 \leq x_{1} \leq 0.8,10 \leq x_{2} \leq 40$,

$3000 \leq x_{3} \leq 20000$

Optimal capacity of gas production facilities [11]:

$\operatorname{Min} f(x)=61.8+5.72 x_{1}+0.2623\left[\left(40-x_{1}\right) \ln \left(\frac{x_{2}}{200}\right)\right]^{-0.85}$

$+0.087\left(40-x_{1}\right) \ln \left(\frac{x_{2}}{200}\right)+700.23 x_{2}^{-0.75}$

Subject to: $x_{1} \geq 17.5, x_{2} \geq 200 ; 17.5 \leq x_{1} \leq 40$,

$300 \leq x_{2} \leq 600$.

Numerical results for the real life problems are listed in Table 5. Numerical results show that in terms of average number of generations required to reach the optimum solution, the proposed DE-PSO gave the best results. However in terms of function value all the algorithms gave more or less similar results.

\section{Conclusions}

A hybrid of DE and PSO called DE-PSO is proposed and its performnce is validated on a set of benchmark and real life problems. Numerical results 
show that DE-PSO outperformed the classical DE and PSO and also two recently proposed hybrid version of DE and PSO. Future research will investigate the performance of proposed DE-PSO on constrained optimization problems.

Table 1. Numerical Benchmark Problems

\begin{tabular}{|c|c|c|}
\hline Function & Range & Optimum \\
\hline$f_{1}(x)=\sum_{i=1}^{n}\left(x_{i}^{2}-10 \cos \left(2 \pi x_{i}\right)+10\right)$ & {$[-5.12,5.12]$} & 0 \\
\hline$f_{2}(x)=\sum_{i=1}^{n} x_{i}^{2}$ & {$[-5.12,5.12]$} & 0 \\
\hline$f_{3}(x)=\frac{1}{4000} \sum_{i=0}^{n-1} x_{i}^{2}-\sum_{i=0}^{n-1} \cos \left(\frac{x_{i}}{\sqrt{i+1}}\right)+1$ & {$[-600,600]$} & 0 \\
\hline$f_{4}(x)=\sum_{i=0}^{n-1} 100\left(x_{i+1}-x_{i}^{2}\right)^{2}+\left(x_{i}-1\right)^{2}$ & {$[-30,30]$} & 0 \\
\hline$f_{5}(x)=-\sum_{i=1}^{n} x_{i} \sin \left(\sqrt{\left|x_{i}\right|}\right)$ & {$[-500,500]$} & $-418.9829 * n$ \\
\hline $\begin{array}{c}f_{6}(x)=(0.1)\left\{\sin ^{2}\left(3 \pi x_{1}\right)+\sum_{i=1}^{n-1}\left(\left(x_{i}-1\right)^{2}\left(1+\sin ^{2}\left(3 \pi x_{i+1}\right)\right)\right)\right. \\
\left.+\left(x_{n}-1\right)\left(1+\sin ^{2}\left(2 \pi x_{n}\right)\right)\right\}+\sum_{i=0}^{n-1} u\left(x_{i}, 5,100,4\right)\end{array}$ & {$[-50,50]$} & -1.1428 \\
\hline $\begin{aligned} f_{7}(x)= & \frac{\pi}{n}\left\{10 \sin ^{2}\left(\pi y_{1}\right)+\sum_{i=1}^{n-1}\left(y_{i}-1\right)^{2}\left[1+10 \sin ^{2}\left(y_{i+1} \pi\right)\right]\right. \\
& \left.+\left(y_{n}-1\right)^{2}\right\}+\sum_{i=1}^{n} u\left(x_{i}, 10,100,4\right)\end{aligned}$ & {$[-50,50]$} & 0 \\
\hline$f_{8}(x)=20+e-20 \exp \left(-0.2 \sqrt{\frac{1}{n} \sum_{i=1}^{n} x_{i}^{2}}\right)-\exp \left(\frac{1}{n} \sum_{i=1}^{n} \cos \left(2 \pi x_{i}\right)\right)$ & {$[-32,32]$} & 0 \\
\hline$f_{9}(x)=\left(\sum_{i=0}^{n-1}(i+1) x_{i}^{4}\right)+\operatorname{rand}[0,1]$ & {$[-1.28,1.28]$} & 0 \\
\hline$f_{10}(x)=\max \left|x_{i}\right|, \quad 0 \leq i<n$ & {$[-100,100]$} & 0 \\
\hline$f_{11}(x)=\sum_{i=0}^{n-1}\left|x_{i}\right|+\prod_{i=0}^{n-1}\left|x_{i}\right|$ & {$[-10,10]$} & 0 \\
\hline$f_{12}(x)=\sum_{i=0}^{n-1}\left\lfloor x_{i}+1 / 2\right\rfloor^{2}$ & {$[-100,100]$} & 0 \\
\hline
\end{tabular}

Table 3. Number of times PSO phase is activated

\begin{tabular}{|c|c|c|c|c|c|c|c|c|c|c|c|}
\hline $\mathrm{f}$ & Dim & $\mathrm{N}_{\mathrm{PSO}}$ & $\mathrm{f}$ & Dim & $\mathrm{N}_{\mathrm{PSO}}$ & $\mathrm{f}$ & Dim & $\mathrm{N}_{\mathrm{PSO}}$ & $\mathrm{f}$ & Dim & $\mathrm{N}_{\text {PSO }}$ \\
\hline \multirow{3}{*}{$f 1$} & 30 & 157 & \multirow{3}{*}{$f 7$} & 30 & 142 & \multirow{3}{*}{$f 4$} & 30 & 180 & \multirow{3}{*}{$f 10$} & 30 & 68 \\
\hline & 50 & 146 & & 50 & 143 & & 50 & 180 & & 50 & 137 \\
\hline & 100 & 309 & & 100 & ---- & & 100 & 337 & & 100 & ---- \\
\hline \multirow{3}{*}{$f 2$} & 30 & 189 & \multirow{3}{*}{$f 8$} & 30 & 135 & \multirow{3}{*}{$f 5$} & 30 & 117 & \multirow{3}{*}{$f 11$} & 30 & 174 \\
\hline & 50 & 162 & & 50 & 147 & & 50 & 117 & & 50 & 176 \\
\hline & 100 & 194 & & 100 & 172 & & 100 & 196 & & 100 & 322 \\
\hline \multirow{3}{*}{$f 3$} & 30 & 178 & \multirow{3}{*}{ f9 } & 30 & 35 & \multirow{3}{*}{ f6 } & 30 & 142 & \multirow{3}{*}{$f 12$} & 30 & 63 \\
\hline & 50 & 177 & & 50 & 52 & & 50 & 163 & & 50 & 70 \\
\hline & 100 & 378 & & 100 & 147 & & 100 & 196 & & 100 & 63 \\
\hline
\end{tabular}


Table 2. Comparison results of PSO, DE and DE-PSO for functions $\mathrm{f} 1-\mathrm{f} 12$

\begin{tabular}{|c|c|c|c|c|c|c|c|c|c|}
\hline $\mathrm{f}$ & Dim & PSO & $\mathrm{DE}$ & DE-PSO & $\mathrm{f}$ & Dim & PSO & $\mathrm{DE}$ & DE-PSO \\
\hline \multirow{3}{*}{$f$} & 30 & $\begin{array}{l}37.819 \\
(7.456)\end{array}$ & $\begin{array}{l}2.531 \\
(5.19026)\end{array}$ & $\begin{array}{l}1.6141 \\
(3.885)\end{array}$ & \multirow{3}{*}{$f 7$} & 30 & $\begin{array}{l}0.020733 \\
(0.0528)\end{array}$ & $\begin{array}{l}5.505 \mathrm{e}-13 \\
(0.00000)\end{array}$ & $\begin{array}{l}5.504 \mathrm{e}-13 \\
(0.000)\end{array}$ \\
\hline & 50 & $\begin{array}{l}75.309 \\
(19.559)\end{array}$ & $\begin{array}{l}41.470 \\
(8.805) \\
\end{array}$ & $\begin{array}{l}\mathbf{2 4 . 5 7 8 8} \\
(14.6261)\end{array}$ & & 50 & $\begin{array}{l}1.75361 \\
(2.4126)\end{array}$ & $\begin{array}{l}3.334 \mathrm{e}-13 \\
(1.488 \mathrm{e}-15)\end{array}$ & $\begin{array}{l}3.303 e-13 \\
(0.000)\end{array}$ \\
\hline & 100 & $\begin{array}{l}186.045 \\
(4.939)\end{array}$ & $\begin{array}{l}261.198 \\
(1.64699)\end{array}$ & $\begin{array}{l}251.491 \\
(8.26771)\end{array}$ & & 100 & ---- & ---- & ---- \\
\hline \multirow{3}{*}{$f 2$} & 30 & $\begin{array}{l}3.542 \mathrm{e}-16 \\
(4.26 \mathrm{e}-16)\end{array}$ & $\begin{array}{l}2.551 \mathrm{e}-47 \\
(3.032 \mathrm{e}-47)\end{array}$ & $\begin{array}{l}4.077 \mathrm{e}-48 \\
(1.593 \mathrm{e}-47)\end{array}$ & \multirow{3}{*}{$f 8$} & 30 & $\begin{array}{l}1.026 \mathrm{e}-08 \\
(1.90 \mathrm{e}-08)\end{array}$ & $\begin{array}{l}7.250 \mathrm{e}-15 \\
(7.742 \mathrm{e}-16)\end{array}$ & $\begin{array}{l}\text { 3.697e-15 } \\
\text { 0.000) }\end{array}$ \\
\hline & 50 & $\begin{array}{l}0.004 \\
(0.003) \\
\end{array}$ & $\begin{array}{l}6.5094 \mathrm{e}-48 \\
(4.205 \mathrm{e}-48)\end{array}$ & $\begin{array}{l}2.753 \mathrm{e}-49 \\
(1.401 \mathrm{e}-49)\end{array}$ & & 50 & $\begin{array}{l}0.797 \\
(0.423)\end{array}$ & $\begin{array}{l}1.717 \mathrm{e}-13 \\
(3.865 \mathrm{e}-14)\end{array}$ & $\begin{array}{l}7.250 \mathrm{e}-15 \\
(1.565 \mathrm{e}-15)\end{array}$ \\
\hline & 100 & $\begin{array}{l}0.070 \\
(1.43 \mathrm{e}-03)\end{array}$ & $\begin{array}{l}2.117 \mathrm{e}-39 \\
(3.576 \mathrm{e}-39)\end{array}$ & $\begin{array}{l}1.093 \mathrm{e}-40 \\
(1.769 \mathrm{e}-40)\end{array}$ & & 100 & $\begin{array}{l}2.53057 \\
(1.45432)\end{array}$ & $\begin{array}{l}1.435 \mathrm{e}-14 \\
(1.790 \mathrm{e}-14)\end{array}$ & $\begin{array}{l}1.025 \mathrm{e}-15 \\
(2.501 \mathrm{e}-14)\end{array}$ \\
\hline \multirow{3}{*}{$f 3$} & 30 & $\begin{array}{l}0.0184 \\
(0.023) \\
\end{array}$ & $\begin{array}{l}\mathbf{0 . 0 0 0 0 0} \\
(0.00000)\end{array}$ & $\begin{array}{l}\mathbf{0 . 0 0 0} \\
(0.000)\end{array}$ & \multirow{3}{*}{ f9 } & 30 & $\begin{array}{l}0.508 \\
(0.2508) \\
\end{array}$ & $\begin{array}{l}\mathbf{0 . 0 0 7 4} \\
(0.001)\end{array}$ & $\begin{array}{l}0.0076 \\
(.002) \\
\end{array}$ \\
\hline & 50 & $\begin{array}{l}0.381 \\
(0.173)\end{array}$ & $\begin{array}{l}5.421 \mathrm{e}-20 \\
(0.00000)\end{array}$ & $\begin{array}{l}\text { 5.421e-20 } \\
(0.00000)\end{array}$ & & 50 & $\begin{array}{l}0.147 \\
(0.035)\end{array}$ & $\begin{array}{l}0.012 \\
(0.002)\end{array}$ & $\begin{array}{l}0.011 \\
(0.001)\end{array}$ \\
\hline & 100 & $\begin{array}{l}1.051 \\
(0.618)\end{array}$ & $\begin{array}{l}8.131 \mathrm{e}-19 \\
(1.154 \mathrm{e}-17)\end{array}$ & $\begin{array}{l}1.084 \mathrm{e}-19 \\
(2.493-18)\end{array}$ & & 100 & $\begin{array}{l}0.781 \\
(0.052)\end{array}$ & $\begin{array}{l}0.0531 \\
(0.021)\end{array}$ & $\begin{array}{l}0.0358 \\
(0.015)\end{array}$ \\
\hline \multirow{3}{*}{ f4 } & 30 & $\begin{array}{l}81.273 \\
(41.218) \\
\end{array}$ & $\begin{array}{l}31.1369 \\
(17.1211)\end{array}$ & $\begin{array}{l}24.202 \\
(12.3086)\end{array}$ & \multirow{3}{*}{ f10 } & 30 & $\begin{array}{l}5.357 \\
(3.204) \\
\end{array}$ & $\begin{array}{l}4.239 \mathrm{e}-06 \\
(\mathbf{1 . 3 2 e - 0 6 )}\end{array}$ & $\begin{array}{l}\mathbf{2 . 4 7 4 e - 0 6} \\
(1.440 \mathrm{e}-06)\end{array}$ \\
\hline & 50 & $\begin{array}{l}174.222 \\
(113.635)\end{array}$ & $\begin{array}{l}50.3377 \\
(16.8557) \\
\end{array}$ & $\begin{array}{l}44.741 \\
(1.402)\end{array}$ & & 50 & $\begin{array}{l}17.643 \\
(2.027) \\
\end{array}$ & $\begin{array}{l}0.0005 \\
(0.0004) \\
\end{array}$ & $\begin{array}{l}3.697 \mathrm{e}-05 \\
(7.255 \mathrm{e}-06) \\
\end{array}$ \\
\hline & 100 & $\begin{array}{l}250.681 \\
(24.643)\end{array}$ & $\begin{array}{l}91.2370 \\
(3.82465)\end{array}$ & $\begin{array}{l}91.024 \\
(3.400)\end{array}$ & & 100 & --- & --- & --- \\
\hline \multirow{3}{*}{$f 5$} & 30 & $\begin{array}{l}-10652.33 \\
(663.174)\end{array}$ & $\begin{array}{l}-12534 \\
(54.2753)\end{array}$ & $\begin{array}{l}-\mathbf{- 1 2 5 4 5 . 8} \\
(\mathbf{4 7 . 3 7 5 3})\end{array}$ & \multirow{3}{*}{ fll } & 30 & $\begin{array}{l}2.063 \mathrm{e}-11 \\
(5.853 \mathrm{e}- \\
12)\end{array}$ & $\begin{array}{l}8.915 \mathrm{e}-27 \\
\mathbf{( 3 . 3 4 8 e - 2 7 )}\end{array}$ & $\begin{array}{l}\mathbf{4 . 7 9 2 e - 2 7} \\
(4.523 \mathrm{e}-27)\end{array}$ \\
\hline & 50 & $\begin{array}{l}-16685.7 \\
(372.981)\end{array}$ & $\begin{array}{l}-20818.9 \\
(123.653)\end{array}$ & $\begin{array}{l}-20913.6 \\
(54.2753)\end{array}$ & & 50 & $\begin{array}{l}0.0681 \\
(0.068)\end{array}$ & $\begin{array}{l}1.670 \mathrm{e}-26 \\
(5.592 \mathrm{e}-27)\end{array}$ & $\begin{array}{l}2.320 \mathrm{e}-27 \\
(1.216 \mathrm{e}-27)\end{array}$ \\
\hline & 100 & $\begin{array}{l}-30417.7 \\
(530.050)\end{array}$ & $\begin{array}{l}41898.3 \\
(0.000)\end{array}$ & $\begin{array}{l}41898.3 \\
(0.000)\end{array}$ & & 100 & $\begin{array}{l}1.7619 \\
(0.976)\end{array}$ & $\begin{array}{l}1.779 \mathrm{e}-22 \\
(9.021 \mathrm{e}-26)\end{array}$ & $\begin{array}{l}1.016 \mathrm{e}-22 \\
(1.191 \mathrm{e}-23)\end{array}$ \\
\hline \multirow{3}{*}{ f6 } & 30 & $\begin{array}{l}-1.1384 \\
(0.0052)\end{array}$ & $\begin{array}{l}-1.149356 \\
(1.857 \mathrm{e}-16)\end{array}$ & $\begin{array}{l}-1.150 \\
(0.000)\end{array}$ & \multirow{3}{*}{$f 12$} & 30 & $\begin{array}{l}0.05 \\
(0.217)\end{array}$ & $\begin{array}{l}0.000 \\
(0.000)\end{array}$ & $\begin{array}{l}0.00000 \\
(0.00000)\end{array}$ \\
\hline & 50 & $\begin{array}{l}37.0296 \\
(22.9197)\end{array}$ & $\begin{array}{l}-1.15044 \\
(0.00000)\end{array}$ & $\begin{array}{l}-1.15044 \\
(0.000)\end{array}$ & & 50 & $\begin{array}{l}3.1 \\
(2.507)\end{array}$ & $\begin{array}{l}\mathbf{0 . 0 0 0} \\
(0.000)\end{array}$ & $\begin{array}{l}\mathbf{0 . 0 0 0 0 0} \\
(0.00000) \\
\end{array}$ \\
\hline & 100 & $\begin{array}{l}173.854 \\
(11.330)\end{array}$ & $\begin{array}{l}-1.150 \\
(0.000)\end{array}$ & $\begin{array}{l}-1.150 \\
(0.000) \\
\end{array}$ & & 100 & $\begin{array}{l}63.5 \\
(4.5)\end{array}$ & $\begin{array}{l}0.000 \\
(0.000)\end{array}$ & $\begin{array}{l}\mathbf{0 . 0 0 0 0 0} \\
(\mathbf{0 . 0 0 0 0 0 )}\end{array}$ \\
\hline
\end{tabular}

Table 4. Comparison of DE-PSO with DEPSO [7] and BBDE [6]

\begin{tabular}{|c|l|l|l|l|l|}
\hline f & \multicolumn{1}{|c|}{$\begin{array}{c}\text { DEPSO [7] } \\
\text { Mean (Std) }\end{array}$} & $\begin{array}{c}\text { DE-PSO (This } \\
\text { paper) } \\
\text { Mean (Std) }\end{array}$ & f & $\begin{array}{c}\text { BBDE [6] } \\
\text { Mean (Std Dev) }\end{array}$ & $\begin{array}{c}\text { DE-PSO (This paper) } \\
\text { Mean (Std Dev) }\end{array}$ \\
\hline F1 & $24.216(6.417)$ & $\mathbf{1 . 6 1 4 1}(\mathbf{3 . 8 8 5})$ & F1 & $72.185(3.018)$ & $\mathbf{1 . 6 1 4 1}(\mathbf{3 . 8 8 5})$ \\
\hline F3 & $6.2 \mathrm{e}-16(4.1 \mathrm{e}-16))$ & $\mathbf{0 . 0 0 0}(\mathbf{0 . 0 0 0})$ & F3 & $0.269 \mathrm{e}-01(0.767-02)$ & $\mathbf{0 . 0 0 0}(\mathbf{0 . 0 0 0})$ \\
\hline F5 & $-12547.7(66.25)$ & $\mathbf{- 1 2 5 5 4 . 3}(95.304)$ & F4 & $\mathbf{1 4 . 2 9 5}(0.948)$ & $24.202(12.3086)$ \\
\hline F7 & $\mathbf{3 . 9 e - 2 0}(4.1 \mathrm{e}-21)$ & $5.505 \mathrm{e}-013(0.000)$ & F8 & $2.1361(0.159)$ & $\mathbf{3 . 6 9 7 e - 0 1 5 ( 0 . 0 0 0 )}$ \\
\hline F8 & $-0.0002(0.0002)$ & $\mathbf{3 . 6 9 7 e - 0 1 5}(\mathbf{0 . 0 0 0})$ & F12 & $0.000(0.000)$ & $\mathbf{0 . 0 0 0}(\mathbf{0 . 0 0 0})$ \\
\hline
\end{tabular}


Table 5. Numerical results of Real Life Problems

\begin{tabular}{|c|c|c|c|c|}
\hline \multicolumn{5}{|c|}{ Gas Transmission Compressor Design } \\
\hline Item & PSO & $\mathrm{DE}$ & $\begin{array}{l}\text { DE- } \\
\text { PSO }\end{array}$ & [11] \\
\hline $\mathrm{x}_{1}$ & 55 & 51.9857 & 53.4474 & 55 \\
\hline $\mathrm{x}_{2}$ & 1.19541 & 1.18335 & 1.1901 & 1.195 \\
\hline $\mathrm{x}_{3}$ & 24.7749 & 24.7195 & 24.7186 & 25.026 \\
\hline$f(x)$ & $\begin{array}{l}296.446 \\
e+004\end{array}$ & $\begin{array}{l}296.448 \\
\mathrm{e}+004\end{array}$ & $\begin{array}{l}296.436 \\
\text { e+004 }\end{array}$ & $\begin{array}{l}296.45 \\
5 \\
e+004\end{array}$ \\
\hline $\mathrm{G}_{\mathrm{Avg}}$ & 786.7 & 146.4 & 129.6 & NA \\
\hline $\mathrm{f}_{\text {Eval }}$ & 23631 & 4422 & 6205.1 & NA \\
\hline $\mathrm{N}_{\mathrm{PSO}}$ & NA & NA & 34 & NA \\
\hline \multicolumn{5}{|c|}{$\begin{array}{l}\text { Optimal Thermohydralic Performance of an } \\
\text { Artificially Roughened Air Heater }\end{array}$} \\
\hline Item & PSO & $\mathrm{DE}$ & $\begin{array}{l}\text { DE- } \\
\text { PSO }\end{array}$ & [12] \\
\hline $\mathrm{x}_{1}$ & $\begin{array}{l}0.0580 \\
9\end{array}$ & 0.12469 & 0.15301 & 0.052 \\
\hline $\mathrm{x}_{2}$ & 10 & 10 & 10 & 10 \\
\hline $\mathrm{x}_{3}$ & $\begin{array}{l}10400 . \\
2\end{array}$ & 3811.07 & 3000 & 10258 \\
\hline$f(x)$ & $\begin{array}{l}4.2142 \\
2\end{array}$ & 4.21422 & 4.21422 & 4.182 \\
\hline $\mathrm{G}_{\mathrm{Avg}}$ & 205.9 & 87.4 & 83.9 & NA \\
\hline $\mathrm{f}_{\text {Eval }}$ & 6207 & 2652 & 4115 & NA \\
\hline $\mathrm{N}_{\text {PSO }}$ & NA & NA & 16 & NA \\
\hline \multicolumn{5}{|c|}{ Optimal Capacity of Gas Production Facilities } \\
\hline Item & PSO & $\mathrm{DE}$ & $\begin{array}{l}\text { DE- } \\
\text { PSO }\end{array}$ & [11] \\
\hline $\mathrm{x}_{1}$ & 17.5 & 17.5 & 17.5 & 17.5 \\
\hline $\mathrm{x}_{2}$ & 600 & 600 & 600 & 465 \\
\hline$f(x)$ & $\begin{array}{l}169.84 \\
4\end{array}$ & 169.844 & 169.844 & 173.76 \\
\hline $\mathrm{G}_{\mathrm{Avg}}$ & 10.4 & 15.1 & 9.9 & NA \\
\hline $\mathrm{f}_{\text {Eval }}$ & 342 & 483 & 423 & NA \\
\hline $\mathrm{N}_{\mathrm{PSO}}$ & NA & NA & 44 & NA \\
\hline
\end{tabular}

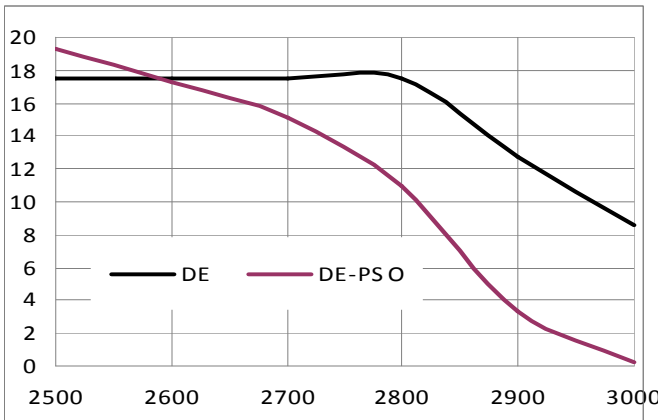

Figure 1(b)

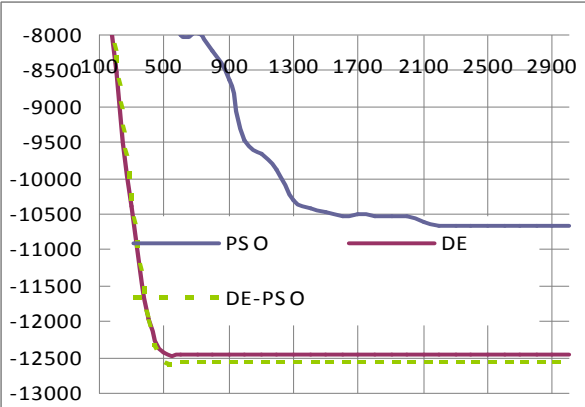

Figure 2(a)

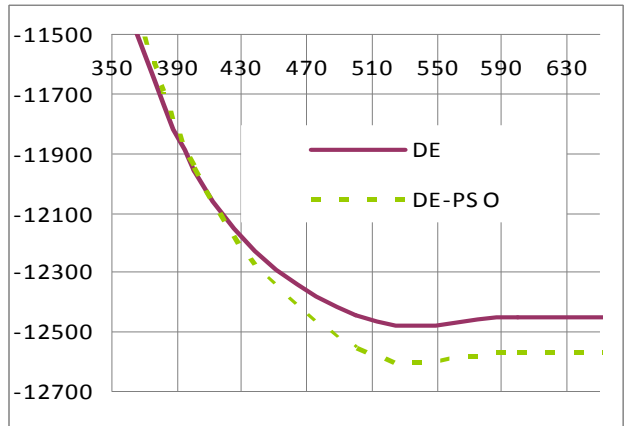

Figure 2 (b)

$\mathrm{G}_{\mathrm{avg}}-$ Average number of generations

$\mathrm{N}_{\text {feval }}$ - number of function evaluations

$\mathrm{N}_{\mathrm{PSO}}$ - Number of times PSO activated

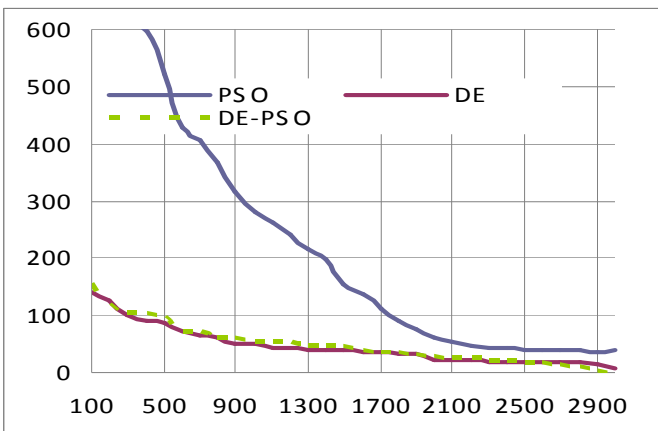

Figure 1(a)

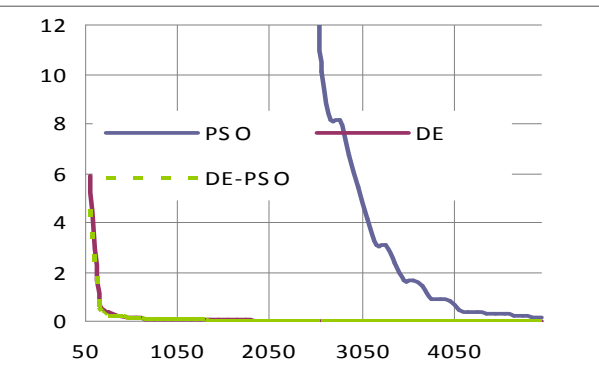

Figure 3 (a) 


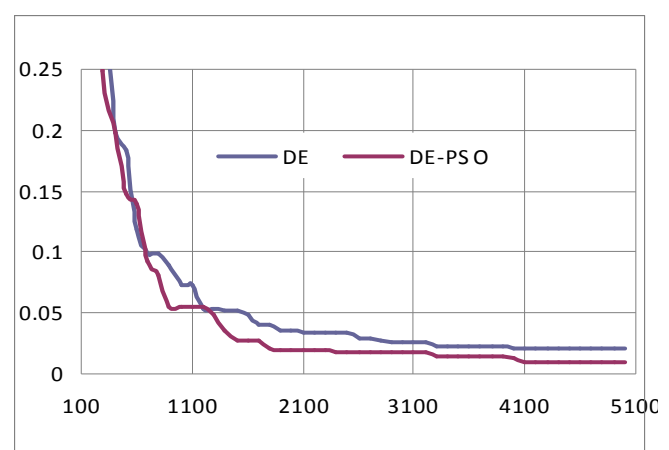

Figure 3 (b)

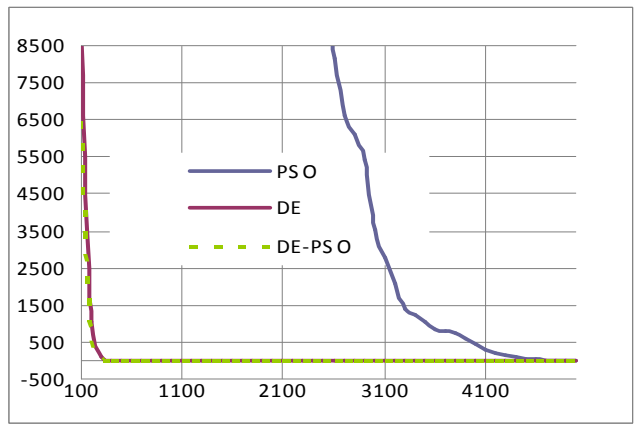

Figure 4(a)

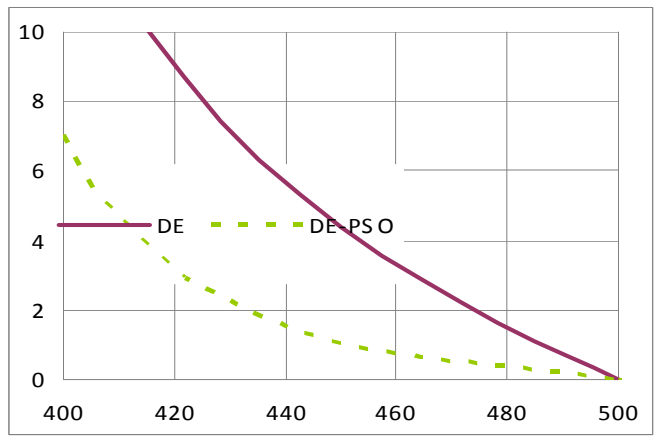

Figure 4 (b)

Figures 1(a) - 4(a) : Performance comparison of DEPSO with DE and PSO for functions f1, f5, f9 and f12 Figures 1(b) - 4(b) : Performance comparison of DEPSO with DE f1, f5, f9 and f12 for the last 1000 generations. In all the above figures, the horizontal axis represents the generation and the vertical axis represents the fitness function value

\section{References}

[1] H. Liu, A. Abraham and W. Zhang, "A Fuzzy Adaptive Turbulent Particle Swarm Optimization", International Journal of Innovative Computing and Applications, Volume 1, Issue 1, 2007, pp. 39-47.
[2] Hendtlass, T., "A Combined Swarm differential evolution algorithm for optimization problems', fourteenth international conference on industrial and engineering applications of artificial intelligence and expert systems, Lecture notes in computer Science, Volume 2070, 11 - 18, Springer Verlag, 2001.

[3] Kannan, S., Slochanal, S.M.R., Subbaraj, P., Padhy, N.P., " Applications of Particle swarm optimization techniques and its variants to generation expansion planning. Electric Power Systems Research, 70 (3); 203-210, 2004.

[4] Zhang, W-T and Xie, X-F, “ DEPSO: hybrid Particle Swarm with Differential Evolution Operator, IEEE International Conference on Systems Man and Cybernetics, Volume 4, pp. 3816 - 3821, 2003.

[5] Talibi, H., and Bautouche "Hybrid Partocle Swarm with Differential Evolution for Multimodal Image Regression", IEEE International Conference on Industrial Technology, Volume 3, pp. 1567-1573, 2004.

[6] Omran, Mohd. G.H., Engelbrecht, A. P., Salman, Ayed, "Differential Evolution based Particle Swarm Optimization", IEEE Swarm Intelligence Symposium (SIS 2007), 2007, pp. $112-119$.

[7] Hao, Z-F, Gua, G-H, Huang, H., "A Particle Swarm Optimization Algorithm with Differential Evolution", Sixth International conference on Machine Learning and Cybernetics, pp. 1031 - 1035, 2007.

[8] R. Storn and K. Price, "Differential Evolution - a simple and efficient Heuristic for global optimization over continuous spaces", Journal Global Optimization. 11, 1997, pp. 341 - 359.

[9] Goldberg, D., "Genetic Algorithms in Search Optimization and Machine Learning," Addison Wesley Publishing Company, Reading, Massachutes.

[10] Kennedy, J. and Eberhart, R., "Particle Swarm Optimization," IEEE International Conference on Neural Networks (Perth, Australia), IEEE Press, 1995, pg. IV: 1942-1948.

[11]Beightler C. S. and Phillips DT, "Applied Geometric Programming", Jhon Wiley and sons, New York, 1976.

[12] Prasad and Saini, "Optimal Thermo Hydraulic Performance of Artificially Roughened Solar Air Heaters", J. Solar Energy, 1991, pp. 91 96. 